\title{
Interleukin-13 and its receptors in colorectal cancer (Review)
}

\author{
RU ZHOU $^{1 *}$, SHIGUANG QIAN $^{2 *}$, XIAODONG GU ${ }^{1}$, ZONGYOU CHEN $^{1}$ and JIANBIN XIANG \\ ${ }^{1}$ Department of General Surgery, Huashan Hospital, Fudan University, Shanghai 200040, P.R. China; \\ ${ }^{2}$ Department of Immunology and General Surgery, Lerner Research Institute, \\ Cleveland Clinic Foundation, Cleveland, OH 44195, USA
}

Received May 05, 2013; Accepted July 08, 2013

DOI: $10.3892 /$ br.2013.132

\begin{abstract}
Interleukin (IL)-13 is an immunoregulatory cytokine secreted by numerous immune cells. Its functions are similar to those of IL-4 and they share a common receptor. This cytokine has been included in recent studies on human tumors and malignant diseases, evoking a scientific interest to investigate the role of IL-13 and its receptors as novel biomarkers and targets for therapy. Colorectal cancer is one of the most common human malignancies, its prognosis is not promising and the efficacy of molecular-targeted therapy has not been established. This review summarizes the currently available data on the role of IL-13 and its receptors in colorectal cancer, including the signaling pathways involved in mediating the effects of IL-13, the role of IL-13 and/or its receptors in the prediction of cancer and several drugs targeting IL-13 or its receptors that are currently under evaluation.
\end{abstract}

\section{Contents}

1. Introduction

2. IL-13-related pathways in cancer surveillance

3. IL-13 in cancer prediction

4. Targeting IL-13 and its receptors in cancer treatment

5. Conclusion

\section{Introduction}

Interleukin (IL)-13 was identified in 1989 (1). Since then, scientists have extensively investigated its structure, biological characteristics and mechanisms of action. IL-13 exhibits a wide range of biological activities and potential clinical applications.

Correspondence to: Professor Zongyou Chen, Department of General Surgery, Huashan Hospital, 12 Middle Wulumuqi Road, Shanghai, Shanghai 200040, P.R. China

E-mail: zhru0456168@gmail.com

*Contributed equally

Key words: colorectal cancer, interleukin-13, interleukin-13 receptor
The location of the IL-13 gene is in close proximity to the IL-4 gene (2). IL-13 is produced by T and B cells, mast cells, basophils, natural killer and dendritic cells (3) and plays a key role in inflammation and immune responses (4). Over the last few years, the known effector functions of IL-13 were significantly expanded (5). This function may be attributed to the development of unique animal models that either promote or inhibit the activity of IL-13 and/or its related gene, including mice deficient in IL-4, IL-4 receptor (R), signal transducer and activator of transcription (STAT) 6 or IL-13, as well as IL-13 transgenic mice that overexpress IL-13 and mice simultaneously deficient in IL-4 and IL-13. In addition, novel IL-13 antagonists and neutralizing antibodies (Abs) have also been generated. The effector functions mediated by IL-13 include the regulation of gastrointestinal parasite expulsion, airway hyperresponsiveness, allergic inflammation, immunoglobulin E Ab production, mastocytosis, goblet cell hyperplasia, tissue eosinophilia, intracellular parasitism, tissue remodeling, fibrosis and tumor cell growth. Some of its effects are also associated with tumor invasion and metastasis.

The receptor components of IL-13 are similar to those of IL-4. This may explain their biological characteristics being similar in many respects. Type I IL-4R only binds IL-4, whereas type II IL-4R binds IL-4 and IL-13 (6). Type II IL-4R is composed of the IL-4R $\alpha$ and IL-13R $\alpha 1$ chains (7). The IL-4R $\alpha$ chain binds IL-4 and the IL-13R $\alpha 1$ chain binds IL-13 (8). IL-13R $\alpha 1$ alone exhibits low affinity for IL-13; however, when IL-4R $\alpha$ and IL-13R $\alpha 1$ combine to form the type II IL-4R dimer, it binds IL-13 with high affinity (9) and the complex mediates signal transduction through the JAK/STAT6 pathway (10). Apart from IL-13R $\alpha 1$, IL-13 has another cognate receptor, IL-13R $\alpha 2$, which binds IL-13 with a markedly high affinity, although it lacks any significant cytoplasmic domain and, therefore, does not mediate signal transduction $(11,12)$. It was reported that IL-13R $\alpha 2$ may regulate IL-13 and IL-4 signal transduction through internalization of IL-13 (13) and that the extracellular domain of IL-13R $\alpha 2$ may serve as a decoy receptor for IL-13 $(14,15)$.

IL-13R $\alpha 2$ is highly expressed in several human tumors. It was previously demonstrated that it may serve as a biomarker of cancer as well as a therapeutic target (16). The prognosis of colorectal cancer, one of the most common human malignancies, is not promising, particularly in cases with distant organ metastasis, although significant advances have been achieved by molecular-targeted therapy and immunotherapy. At present, 
cetuximab and bevacizumab are the main molecular-targeted drugs used in the treatment of colorectal cancer. However, recent studies indicated that not all colorectal cancer patients benefit from cetuximab therapy. Therefore, there is a need for more effective molecular targets and other therapeutic approaches $(17,18)$. IL-13R $\alpha 2$ may be an important target of immunotherapy in colorectal cancer and recombinant IL-13R $\alpha 2$ protein may also be used as an antitumor vaccine, which may provide novel strategies for the treatment of colorectal cancer.

\section{IL-13-related pathways in cancer surveillance}

As mentioned above, IL-13Rs share components with IL-4Rs. Based on previous experiments conducted on colon carcinoma cell lines, IL-4 and IL-13 also share numerous signaling events. The ligation of IL-13 and IL-4R2 leads to rapid phosphorylation of three out of the four members of the Janus family of kinases (JAKs). Murata et al demonstrated that, within 1 min of activation, JAK2 was phosphorylated, with a peak at $10 \mathrm{~min}$. IL-13 also promoted the phosphorylation of insulin response substrate-1, IL-4R p140, JAK1 and tyrosine kinase 2, but not that of JAK3 kinase. IL-4 also stimulates these kinases and substrates; however, it does not include JAK3 activation in its signaling in colon cancer cell lines. IL-4 and IL-13 induce the phosphorylation of STAT6, but not that of STAT1, STAT3 or STAT5. Data from that study suggested that IL-13 utilizes IL-4R and its signaling pathways and JAK2 may play an important role in the function of IL-4R and IL-13R in colon cancer cells (19). Kawakami et al (11) demonstrated that IL-13 did not activate STAT6 in IL-13R $\alpha 2$-transfected cells, although it efficiently induced activation of the STAT6 protein in cells transfected with the IL-13R $\alpha 1$ and IL-4R $\alpha$ chains, whereas transfection with the IL-13R $\alpha 2$ chain inhibited this activation (11). It has been generally accepted that chronic inflammation in ulcerative colitis is a significant risk factor for colorectal cancer. IL-13 is elevated in the ulcerative colitis mucosa and may induce deregulated signaling in neighboring intestinal epithelial cells; therefore, IL-13 may be considered a tumorigenic cytokine. In addition, IL-13R $\alpha 2$ as a decoy receptor may also participate in modulating the balance and intensity of the signaling pathways initiated in ulcerative colitis and colorectal cancer (20).

IL-13-associated pathways are also involved in the development of other tumors. For example, Fujisawa et al (21) assessed cell invasion and protease activity in ovarian tumor cell lines that overexpress or are deficient in IL-13R $\alpha 2$ and observed that IL-13 treatment significantly augmented cell invasion and enzyme activities in IL-13R $\alpha 2$-positive but not in IL-13R $\alpha 2$-negative cells in vitro. Mechanistically, IL-13 enhances the extracellular-signal-regulated kinase (ERK) $1 / 2$, transcription factor AP-1 and matrix metalloproteinase (MMP) activities in IL-13R $\alpha 2$-positive but not in IL-13R $\alpha 2$-negative cells. By contrast, other signaling pathways, such as the insulin receptor substrate-1 and -2, phosphoinositide 3-kinase and AKT pathways are involved in IL-13 signaling in ovarian cancer cell lines. This was confirmed by the fact that highly specific inhibitors for MMP and AP-1 efficiently inhibited invasion and protease activities in IL-13R $\alpha 2$-positive cells without affecting the basal level invasion and protease activities in vitro (22).
IL-13R $\alpha 2$ has been widely recognized to be a secreted receptor that is devoid of signaling activity and functions only as a decoy receptor that delays signaling via IL-13R $\alpha 1$. An important role of IL-13 signaling via IL-13R $\alpha 2$ may be in tumor immune surveillance. Thus, relevant studies demonstrated that NKT cells stimulated by tumor antigens produce IL-13, which subsequently acts on Gr- $1^{+}$cells to induce the expression of transforming growth factor- $\beta 1$ (TGF- $\beta 1$ ); the latter then inhibits $\mathrm{CD} 8^{+} \mathrm{T}$ cells involved in tumor immune surveillance (23).

\section{IL-13 in cancer prediction}

Considering that cytokines are the key components in the inflammatory microenvironment, a better understanding of colorectal cancer at the molecular level may provide important tools for genetic screening in high-risk groups. A proinflammatory genotype is positively associated with colorectal adenoma, a precursor of colorectal cancer. In a previous study, an analysis of the IL-13 gene with polymerase chain reaction-restriction fragment length polymorphism (PCR-RFLP) was conducted on a Polish population comprising 150 cancer patients and 170 healthy subjects. The results of that study suggested the possibility of an association between IL-13 gene polymorphism and colorectal cancer risk (24). Gunter et al (25) investigated the association of colorectal adenoma with 19 single-nucleotide polymorphisms in a range of important proinflammatory cytokines (IL-1B, IL-6, IL-8, TNF and LTA), anti-inflammatory cytokines (IL-4, IL-10 and IL-13) and the inflammation-related genes PTGS2 and PPARG in a case-control study of risk factors for colorectal polyps. The exploratory data obtained from that study provided evidence that polymorphic variations in genes that regulate inflammation may affect the risk for colorectal adenoma (25).

Inflammatory mediators are also of considerable interest as potential therapeutic targets in various types of cancer. IL-4R $\alpha$ protein expression has been observed in human colon adenocarcinomas (26). In order to determine the role of IL-4R $\alpha$ in colonic tumorigenesis, human and murine colon tumor cell lines expressing IL-4R $\alpha$ were investigated. Treatment with exogenous ligands resulted in dose-dependent increases in proliferation. Mice with global deletion of IL-4R $\alpha$ developed significantly fewer and smaller tumors. A systemic blockade of IL-4 and IL-4R $\alpha$ interactions achieved similar results, suggesting that IL-4R $\alpha$ ligation may contribute to tumor formation in mouse models (27).

A flow cytometric analysis revealed that monoclonal antibodies (mAbs) directed against E-cadherin and carcinoembryonic antigen (CEA) inhibited Colo 205 cell-cell adhesion. IL-4 significantly inhibited the expression of E-cadherin and CEA (26) and IL-13 inhibited colon cancer cell-cell adhesion. These results suggested that IL-4 and IL-13 inhibit colon cancer cell-cell adhesion by downregulating the expression of the adhesion molecules E-cadherin and CEA. In an animal model of human ovarian cancer, IL-13R $\alpha 2$-positive tumors metastasized to the lymph nodes and peritoneum earlier during the course of the disease compared to IL-13R $\alpha 2$-negative tumors. Of note, the IL-13R $\alpha 2$-positive tumor-bearing mice died earlier compared to the mice with IL-13R $\alpha 2$-negative tumors. Intraperitoneal injection of IL-13 further shortened 
the survival of IL-13R 2 2-positive tumor-bearing mice compared to IL-13R $\alpha 2$-negative ones. IL-13R $\alpha 2$-positive tumors and lymph node metastasis were associated with higher levels of MMPs and ERK1/2 expression compared to IL-13R $\alpha 2$-negative tumors (23).

In a mouse model with a conditional deletion of TGF- $\beta$ signaling in the neurons, $35 \%$ of the mice developed spontaneous squamous cell carcinomas. Transplantation of these tumors into athymic nude mice resulted in $62 \%$ tumorigenicity. A total of $33 \%$ of the tumors expressed the IL-13R $\alpha 2$ chain (30).

\section{Targeting IL-13 and its receptors in cancer treatment}

The studies on IL-13 and IL-13-related receptors as therapeutic targets have been mainly focused on asthma and allergic diseases $(27,28,29,31,32)$ with substantial achievements (Table I).

The immunotherapeutic approaches including cancer therapeutic vaccines targeting specific tumor-associated antigens are currently at various stages of development. The IL-13R $\alpha 2$-directed cancer vaccine may be one of the most promising approaches to tumor immunotherapy, due to the selective expression of IL-13R $\alpha 2$ in various solid tumor types and its absence in normal tissues. Nakashima et al (33) provided experimental evidence that the IL-13R $\alpha 2$ chain was a functional component of an IL-13R system that promotes binding and internalization of the ligand. In addition, the IL-13R $\alpha 2$ chain interacts with the IL-4R $\alpha$ chains to inhibit the effect of IL-13 on STAT6 activation. IL-13R $\alpha 2$ is not commonly expressed by normal immune and non-immune cells. These observations suggested that IL-13R $\alpha 2$ is a potential target for receptor-directed immunotherapy or gene cancer therapy.

The studies on IL-13R $\alpha 2$-related cancer treatment have been mainly performed on brain tumors. Hsi et al (34) knocked down IL-13R $\alpha 2$ with short interfering RNA and markedly promoted tumor cell apoptosis and reduced glioblastoma multiforme tumor growth in vivo. The authors of that study identified a mechanism for eliminating the blockade of endogenous IL-13 signaling to promote tumor cell apoptosis. This targeting of IL-13R $\alpha 2$ may be a novel approach to pharmacological intervention in brain tumors (34). A previous USA study (35) demonstrated that IL-13R $\alpha 2$ was expressed by glioma stem-like cancer-initiating cells and differentiated tumor populations, rendering them targetable by IL-13R $\alpha 2$-specific human $\mathrm{CD}^{+}$cytotoxic T cells (CTLs) [IL-13-zetakine ${ }^{+}$CTLs (36). Wang et al synthesized IL-13 cDNA de novo by PCR, the cDNA was ligated into the plasmid vector and the $\mathrm{T}$ cells were transfected. This receptor recognized IL-13R $\alpha 2$ and initiated cytolytic killing. It also incorporated a point mutation in the IL-13 ligand domain for preferential binding to IL-13R $\alpha 2$ and reduced affinity to IL-13R $\alpha 1$. Moreover, in an ovarian cancer animal model, IL-13R $\alpha 2$ was shown to be involved in cancer metastasis through the activation of ERK/AP-1 pathway. Therefore, targeting IL-13R $\alpha 2$ may not only directly kill primary tumors but also prevent cancer metastasis (23).

Although there is no direct evidence supporting the use of IL-13R $\alpha 2$ in the treatment of colorectal cancer, the IL-13 regulatory pathway of tumor immune surveillance appeared to
Table I. mAbs and fusion proteins under development for asthma or allergic diseases ${ }^{\mathrm{a}}$.

\begin{tabular}{lll}
\hline Name & \multicolumn{1}{c}{ Source } & Target \\
\hline AMG-317 & Human & IL-4R $\alpha$ \\
Pitrakinra & Mutein & IL-4R $\alpha$ \\
CAT-354 & Human & IL-13 \\
Lebrikizumab & Humanized & IL-13 \\
QAX-576 & Human mAb & IL-13 \\
IL-13E13K & Mutein & IL-13 \\
GM1E7 & Mouse mAb & IL-13 1 \\
\hline
\end{tabular}

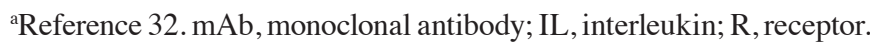

be active in a murine colon carcinoma lung metastasis model, in which tumor growth was partially inhibited in mice treated with an IL-13 inhibitor (5). A previous study conducted by Sinha et al also supported IL-4R $\alpha$ as a therapeutic target in colon cancer (26). Although TGF- $\beta$ inhibition enhanced antitumor immunity mediated by $\mathrm{CD}^{+} \mathrm{T}$ cells in several tumor models, anti-TGF- $\beta$ mAb alone did not exhibit any antitumor effects. Experimental data provided by Shun et al indicated that anti-TGF- $\beta$ enhanced the efficacy of a prophylactic vaccine in normal individuals, despite the absence of the elevated TGF- $\beta$ levels observed in cancer patients (27).

In August, 2012, a novel mAb against IL-13R $\alpha 2$, which competes with IL-13 for binding to IL-13R $\alpha 2$ with high specificity and affinity, was generated and characterized (35). Therefore, studies assessing the therapeutic and diagnostic properties of this $\mathrm{mAb}$ in tumors are possible in the future. IL-13 may also be a potential predictive marker for cancer treatment, since the biological basis of tumor response to radiotherapy (RT) and chemotherapy (CT) is associated not only with the tumor and its microenvironment, but also with the host genetic background. The genetic polymorphisms are potential prognostic factors in several types of cancer. A previous study (37) that investigated a total of 128 SNPs distributed over 76 genes, demonstrated that IL-13 rs1800925 polymorphism is an independent predictive marker of the response to preoperative RT-CT in rectal cancer. Such markers may help discriminate rectal cancer patients who may benefit from neoadjuvant RT-CT from those who are candidates for targeted therapy (cetuximab or bevacizumab). Identification of IL-13 rs1800925 may allow personalization of rectal cancer therapy with a single blood sample test.

\section{Conclusion}

The advances in cancer treatment research are currently focused on the identification of new targets and the corresponding targeting drugs. However, the majority of these drugs did not withstand the test of time and repeated verification. The environment and signal transduction pathways in the human body are complex network systems and the various foreign interventions are mutually influent. In traditional chemotherapy, the key mechanistic step may be ultimately identified as blocking a signaling pathway, inhibiting the expression of a 
receptor, or affecting the binding of the ligand to the receptor, whereas the ultimate mechanism of novel targeted drugs may be identical to the currently available traditional therapies. Therefore, it is recommended that the focus is shifted from the design of novel molecular targeting drugs to the elucidation of the mechanisms underlying tumor occurrence, development and metastasis, which, in itself, may lead to the overcoming of several problems.

\section{Acknowledgements}

This study was supported by Shanghai Young Doctor Training Plan.

\section{References}

1. Brown KD, Zurawski SM, Mosmann TR, et al: A family of small inducible proteins secreted by leukocytes are members of a new superfamily that includes leukocyte and fibroblast-derived inflammatory agents, growth factors, and indicators of various activation processes. J Immunol 142: 679-687, 1989.

2. Smirnov DV, Smirnova MG, Korobko VG, et al: Tandem arrangement of human genes for interleukin-4 and interleukin-13: resemblance in their organization. Gene 155: 277-281, 1995.

3. Terabe M, Park JM and Berzofsky JA: Role of IL-13 in regulation of anti-tumor immunity and tumor growth. Cancer Immunol Immunother 53: 79-85, 2004.

4. Joshi BH, Hogaboam C, Dover P, et al: Role of interleukin-13 in cancer, pulmonary fibrosis, and other $\mathrm{T}(\mathrm{H}) 2$-type diseases Vitam Horm 74: 479-504, 2006.

5. Wynn TA: IL-13 effector functions. Annu Rev Immunol 21: 425-456, 2003

6. Obiri NI, Debinski W, Leonard WJ, et al: Receptor for interleukin 13. Interaction with interleukin 4 by a mechanism that does not involve the common gamma chain shared by receptors for interleukins 2, 4, 7, 9, and 15. J Biol Chem 270: 8797-8804, 1995.

7. Aman MJ, Tayebi N, Obiri NI, et al: cDNA cloning and characterization of the human interleukin 13 receptor alpha chain J Biol Chem 271: 29265-29270, 1996.

8. Zurawski SM, Vega F Jr, Huyghe B, et al: Receptors for interleukin-13 and interleukin-4 are complex and share a novel component that functions in signal transduction. EMBO J 12 : 2663-2670, 1993.

9. Hilton DJ, Zhang JG, Metcalf D, et al: Cloning and characterization of a binding subunit of the interleukin 13 receptor that is also a component of the interleukin 4 receptor. Proc Natl Acad Sci USA 93: 497-501, 1996.

10. O'Shea JJ, Gadina M and Schreiber RD: Cytokine signaling in 2002: new surprises in the Jak/Stat pathway. Cell 109 (Suppl): S121-S131, 2002.

11. Kawakami K, Taguchi J, Murata T, et al: The interleukin-13 receptor alpha 2 chain: an essential component for binding and internalization but not for interleukin-13-induced signal transduction through the STAT6 pathway. Blood 97: 2673-2679, 2001

12. Zhang JG, Hilton DJ, Willson TA, et al: Identification, purification, and characterization of a soluble interleukin (IL)-13-binding protein. Evidence that it is distinct from the cloned Il-13 receptor and Il-4 receptor alpha-chains. J Biol Chem 272: 9474-9480, 1997.

13. Andrews AL, Nasir T, Bucchieri F, et al: IL-13 receptor alpha 2: a regulator of IL-13 and IL-4 signal transduction in primary human fibroblasts. J Allergy Clin Immunol 118: 858-865, 2006.

14. Chiaramonte MG, Mentink-Kane M, Jacobson BA, et al: Regulation and function of the interleukin 13 receptor alpha 2 during a T helper cell type 2-dominant immune response. J Exp Med 197: 687-701, 2003.

15. Rahaman SO, Sharma P, Harbor PC, et al: IL-13R(alpha)2, a decoy receptor for IL-13 acts as an inhibitor of IL-4-dependent signal transduction in glioblastoma cells. Cancer Res 62 : 1103-1109, 2002

16. Fujisawa T, Joshi B, Nakajima A, et al: A novel role of interleukin-13 receptor alpha2 in pancreatic cancer invasion and metastasis. Cancer Res 69: 8678-8685, 2009.
17. Alberts SR, Sargent DJ, Nair S, et al: Effect of oxaliplatin, fluorouracil, and leucovorin with or without cetuximab on survival among patients with resected stage III colon cancer: a randomized trial. JAMA 307: 1383-1393, 2012.

18. Tveit KM, Guren T, Glimelius B, et al: Phase III trial of cetuximab with continuous or intermittent fluorouracil, leucovorin, and oxaliplatin (Nordic FLOX) versus FLOX alone in first-line treatment of metastatic colorectal cancer: the NORDIC-VII study. J Clin Oncol 30: 1755-1762, 2012.

19. Murata T, Noguchi PD and Puri RK: IL-13 induces phosphorylation and activation of JAK2 Janus kinase in human colon carcinoma cell lines: similarities between IL-4 and IL-13 signaling. J Immunol 156: 2972-2978, 1996.

20. Mandal D and Levine AD: Elevated IL-13Ralpha2 in intestinal epithelial cells from ulcerative colitis or colorectal cancer initiates MAPK pathway. Inflamm Bowel Dis 16: 753-764, 2010.

21. Fujisawa T, Joshi BH and Puri RK: IL-13 regulates cancer invasion and metastasis through IL-13R $\alpha 2$ via ERK/AP-1 pathway in mouse model of human ovarian cancer. Int $\mathrm{J}$ Cancer 131: 344-356, 2012.

22. Wang DJ, Brandsma M, Yin Z, et al: A novel platform for biologicaly active recombinant human interleukin-13 production. Plant Biotechnol J 6: 504-515, 2008.

23. Strober W, Kitani A, Fichtner-Feigl S, et al: The signaling function of the IL-13Ralpha2 receptor in the development of gastrointestinal fibrosis and cancer surveillance. Curr Mol Med 9: 740-750, 2009.

24. Walczak A, Przybylowska K, Trzcinski R, et al: Association of $-1112 \mathrm{c} / \mathrm{t}$ promoter region polymorphism of the interleukin 13 gene with occurrence of colorectal cancer. Pol Przegl Chir 83: 27-31, 2011

25. Gunter MJ, Canzian F, Landi S, et al: Inflammation-related gene polymorphisms and colorectal adenoma. Cancer Epidemiol Biomarkers Prev 15: 1126-1131, 2006.

26. Sinha P,Parker KH,Horn L, et al: Tumor-induced myeloid-derived supressor cell function is independent of IFN- $\gamma$ and IL-4R $\alpha$. Eur J Immunol 42: 2052-2059, 2012.

27. Shun MC, Yu W, Gapor A, et al: Pro-apoptotic mechanisms of action of a novel vitamin E analog (alpha-TEA) and a naturally occurring form of vitamin $\mathrm{E}$ (delta-tocotrienol) in MDA-MB-435 human breast cancer cells. Nutr Cancer 48: 95-105, 2004.

28. Koller FL, Hwang DG, Dozier EA, et al: Epithelial interleukin-4 receptor expression promotes colon tumor growth. Carcinogenesis 31: 1010-1017, 2010.

29. Kanai T, Watanabe M, Hayashi A, et al: Regulatory effect of interleukin-4 and interleukin-13 on colon cancer cell adhesion. Br J Cancer 82: 1717-1723, 2000.

30. Honjo Y, Bian Y, Kawakami K, et al: TGF-beta receptor I conditional knockout mice develop spontaneous squamous cell carcinoma. Cell Cycle 6: 1360-1366, 2007.

31. Mentink-Kane MM and Wynn TA: Opposing roles for IL-13 and IL-13 receptor alpha 2 in health and disease. Immunol Rev 202: 191-202, 2004.

32. Mitchell J, Dimov V and Townley RG: IL-13 and the IL-13 receptor as therapeutic targets for asthma and allergic disease. Curr Opin Investig Drugs 11: 527-534, 2010.

33. Nakashima H, Husain SR and Puri RK: IL-13 receptor-directed cancer vaccines and immunotherapy. Immunotherapy 4: 443-451, 2012.

34. Hsi LC, Kundu S, Palomo J, et al: Silencing IL-13R $\alpha 2$ promotes glioblastoma cell death via endogenous signaling. Mol Cancer Ther 10: 1149-1160, 2011.

35. Brown CE, Starr R, Aguilar B, et al: Stem-like tumor-initiating cells isolated from IL-13R $\alpha 2$ expressing gliomas are targeted and killed by IL13-zetakine-redirected T cells. Clin Cancer Res 18: 2199-2209, 2012.

36. Kahlon KS, Brown C, Cooper LJ, et al: Specific recognition and killing of glioblastoma multiforme by interleukin 13-zetakine redirected cytolytic T cells. Cancer Res 64: 9160-9166, 2004.

37. Balyasnikova IV, Wainwright DA, Solomaha E, et al: Characterization and immunotherapeutic implications for a novel antibody targeting interleukin (IL)-13 receptor $\alpha 2$. J Biol Chem 287: 30215-30227, 2012.

38. Ho-Pun-Cheung A, Assenat E, Bascoul-Mollevi C, et al: A large-scale candidate gene approach identifies SNPs in SOD2 and IL13 as predictive markers of response to preoperative chemoradiation in rectal cancer. Pharmacogenomics $\mathrm{J}$ 11: 437-443, 2011. 